\title{
Postoperative pain treatment with erector spinae plane block and pectoralis nerve blocks in patients undergoing mitral/ tricuspid valve repair - a randomized controlled trial
}

\author{
Bogusław Gawęda ${ }^{1}$, Michał Borys²* $2^{*}$, Bartłomiej Belina ${ }^{3}$, Janusz Bąk', Miroslaw Czuczwar²,
}

Bogumiła Wołoszczuk-Gębicka ${ }^{3}$, Maciej Kolowca ${ }^{1}$ and Kazimierz Widenka ${ }^{1}$

\begin{abstract}
Background: Effective postoperative pain control remains a challenge for patients undergoing cardiac surgery. Novel regional blocks may improve pain management for such patients and can shorten their length of stay in the hospital.

To compare postoperative pain intensity in patients undergoing cardiac surgery with either erector spinae plane (ESP) block or combined ESP and pectoralis nerve (PECS) blocks.

Methods: This was a prospective, randomized, controlled, double-blinded study done in a tertiary hospital. Thirty patients undergoing mitral/tricuspid valve repair via mini-thoracotomy were included. Patients were randomly allocated to one of two groups: ESP or PECS + ESP group (1:1 randomization). Patients in both groups received a single-shot, ultrasound-guided ESP block. Participants in PECS + ESP group received additional PECS blocks. Each patient had to be extubated within $2 \mathrm{~h}$ from the end of the surgery. Pain was treated via a patient-controlled analgesia (PCA) pump. The primary outcome was the total oxycodone consumption via PCA during the first postoperative day. The secondary outcomes included pain intensity measured on the visual analog scale (VAS), patient satisfaction, Prince Henry Hospital Pain Score (PHHPS), and spirometry.
\end{abstract}

Results: Patients in the PECS + ESP group used significantly less oxycodone than those in the ESP group: median 12 [interquartile range (IQR): 6-16] mg vs. 20 [IQR: 18-29] mg ( $p=0.0004)$. Moreover, pain intensity was significantly lower in the PECS + ESP group at each of the five measurements during the first postoperative day. Patients in the PECS + ESP group were more satisfied with pain management. No difference was noticed between both groups in PHHPS and spirometry.

Conclusions: The addition of PECS blocks to ESP reduced consumption of oxycodone via PCA, reduced pain intensity on the VAS, and increased patient satisfaction with pain management in patients undergoing mitral/ tricuspid valve repair via mini-thoracotomy.

Trial registration: The study was registered on the 19th July 2018 (first posted) on the ClinicalTrials.gov identifier: NCT03592485.

Keywords: Erector spinae plane (ESP) block, Pectoralis nerve (PECS) blocks, Patient-controlled analgesia (PCA), Visual analog scale (VAS)

\footnotetext{
* Correspondence: michalborys1@gmail.com

${ }^{2}$ Second Department of Anesthesia and Intensive Care, Medical University of

Lublin, ul. Staszica 16, 20-081 Lublin, Poland

Full list of author information is available at the end of the article
}

(c) The Author(s). 2020 Open Access This article is distributed under the terms of the Creative Commons Attribution 4.0 International License (http://creativecommons.org/licenses/by/4.0/), which permits unrestricted use, distribution, and 


\section{Background}

Postoperative pain remains a primary challenge in patients undergoing thoracotomy [1]. Poorly managed postoperative pain is associated with an increased number of postoperative complications, including prolonged mechanical ventilation and pulmonary infections [2, 3]. Well-established pain management is an essential aspect of the Enhanced Recovery After Surgery (ERAS) protocol [4]. Recently, we have attempted to institute the ERAS protocol for cardiac surgery procedures performed in our department. Thus, an effective and safe analgesic technique was needed, which was compatible with the ERAS concept.

Among many regional anesthesia techniques for patients undergoing cardiac surgery, thoracic epidural analgesia (TEA) is associated with reduced incidences of cardiovascular events and infections, lower cost, and shortened length of hospital stay [5-7]. Thoracic paravertebral block (PVB) exhibits similar effectiveness to that of TEA for analgesia after cardiothoracic surgery [8, 9]. Other regional anesthesia techniques are not wellestablished in cardiothoracic surgery [10]. Novel fascial blocks, including the erector spinae plane (ESP) block and pectoralis nerve (PECS) block, have been recently proposed as effective methods of pain management for patients undergoing cardiac surgery $[11,12]$.

Our previous, prospective, cohort study demonstrated that the ESP block combined with low-dose intravenous oxycodone was an effective analgesic technique for patients who had undergone mitral or/and tricuspid valve repair via right mini-thoracotomy [13]. In that study, all patients could be weaned from mechanical ventilation within $2 \mathrm{~h}$ postoperatively and were transferred to the general ward on the second postoperative day. However, an abrupt reduction in pain intensity was observed at the 24th postoperative hour; this was clearly associated with the removal of chest drains. We hypothesized that an additional regional block, covering the area of the anterior part of the chest wall, might improve postoperative pain management $[14,15]$.

The objective of this study was to compare postoperative pain intensity in patients undergoing cardiac surgery with either ESP block or combined ESP and PECS blocks by assessing oxycodone consumption during the first operative day (primary objective), as well as by comparing patients' subjective pain intensity by using the visual-analogue scale (VAS, secondary objective).

\section{Methods}

This was a randomized, controlled, double-blind trial conducted in a tertiary cardiac surgery department. Before patient recruitment, the study protocol was approved by the Bioethics Committee of the Medical University of Lublin, Lublin, Poland (permit number KE-
0254/127/2018), and registered at ClinicalTrials.gov (NCT03592485). Written informed consent was obtained from each patient, and the study was conducted in accordance with the tenets of the Declaration of Helsinki for medical research involving human subjects.

\section{Participants}

The inclusion criteria were as follows: patients who (1) required mitral and/or tricuspid valve repair; (2) underwent surgery via right mini-thoracotomy approach; (3) were more than 18 years of age; and (4) were less than 80 years of age. The exclusion criteria included: (1) coagulopathy, defined as known bleeding disorder; (2) allergy to local anaesthetics; (3) depression, which could significantly influence pain perception; (4) epilepsy; (5) antidepressant or epileptic drug treatment; (6) chronic usage of analgesic drugs; (7) addiction to alcohol or recreational drugs. Data from patients who required endotracheal intubation and respiratory support for $>2 \mathrm{~h}$ from the end of surgery were also excluded from the analysis.

\section{Intervention}

Patients were randomly allocated to one of two groups (1:1 ratio, parallel randomization) via computergenerated randomization conducted by a team member who was not involved in the surgery or patient assessment. The same team member prepared opaque envelopes in which the intervention type was concealed. These envelopes were opened a few minutes before attempting the regional block. Patients were randomly assigned to the ESP or PECS + ESP group.

In the ESP group, ultrasound-guided ESP block at the fourth thoracic level was performed before the surgery and induction of general anesthesia with Ropivacaine (0.375\%; Ropimol, Molteni, Italy, $0.2 \mathrm{~mL} / \mathrm{kg}$ ) as described in our previous study (Fig. 1) [13]. The maximum dosage of ropivacaine could not exceed $20 \mathrm{~mL}$ in this group. In the PECS + ESP group, in addition to ESP block, ultrasound-guided PECS blocks type I and II were performed. Local anesthetic $(6-8 \mathrm{ml})$ was deposited in the fascial plane between the pectoralis major and minor muscles (PECS I, Fig. 2); 12-14 ml was deposited between the pectoralis minor and serratus anterior muscles (PECS II, Fig. 3). The total dose of local anesthetic could not exceed $40 \mathrm{~mL}$ (150 mg of ropivacaine) in this group.

\section{Anesthesia}

Etomidate (Hypnomidate, Janssen-Cilag International NV, Belgium), remifentanil (0.5-1.0 $\mathrm{mcg} \mathrm{kg}^{-1} \mathrm{~min}^{-1}$ ) (Ultiva, GlaxoSmithKline, UK), and rocuronium $\left(0.6 \mathrm{mg} \mathrm{kg}^{-1}\right)$ (Esmeron, N.V. Organon, Holland) were used for the induction of general anesthesia. Maintenance was provided with 0.5 minimum alveolar concentration of sevoflurane (age-adjusted, Sevorane, Abbvie, USA), remifentanil, and 


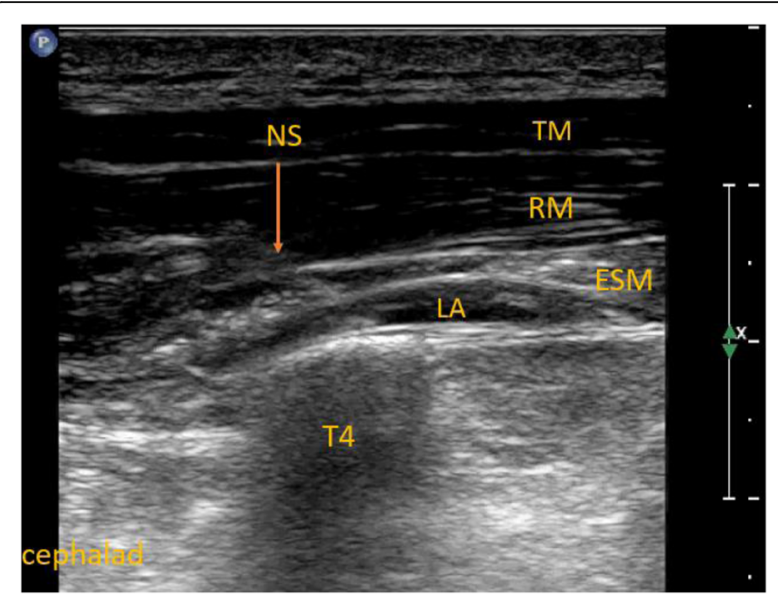

Fig. 1 Erector spinae plane block. ESM - erector spinae muscle, LA local anesthetic, NS- needle shaft, RM- rhomboid muscle, T4 - the transverse process of the fourth thoracic vertebra, TM trapezius muscle

incremental doses of rocuronium. Remifentanil was continued to achieve a target plasma concentration of 4-8 ng $\mathrm{ml}^{-1}$ and adjusted to the patient's heart rate and blood pressure. During the procedure, the right lung was deflated, and the left lung was ventilated with a mixture of air and $\mathrm{O}_{2}$. Residual neuromuscular block was reversed with sugammadex (BridionN.V. Organon, Holland) at the end of surgery.

An intravenous bolus of oxycodone $\left(0.1 \mathrm{mg} \mathrm{kg}^{-1}\right)$ was administered $30 \mathrm{~min}$ prior to the surgery end. Patients were transferred to the intensive care unit where target plasma concentration of remifentanil was reduced to $0.5-2 \mathrm{ng} \mathrm{ml}^{-1}$. Ventilation was continued for $60-120 \mathrm{~min}$ and patients were observed for occurrence of excessive postoperative



Fig. 2 Pectoralis nerves block type I. LA - local anesthetic, NS needle shaft, PM - pectoralis major muscle, Pm - pectoralis minor muscle

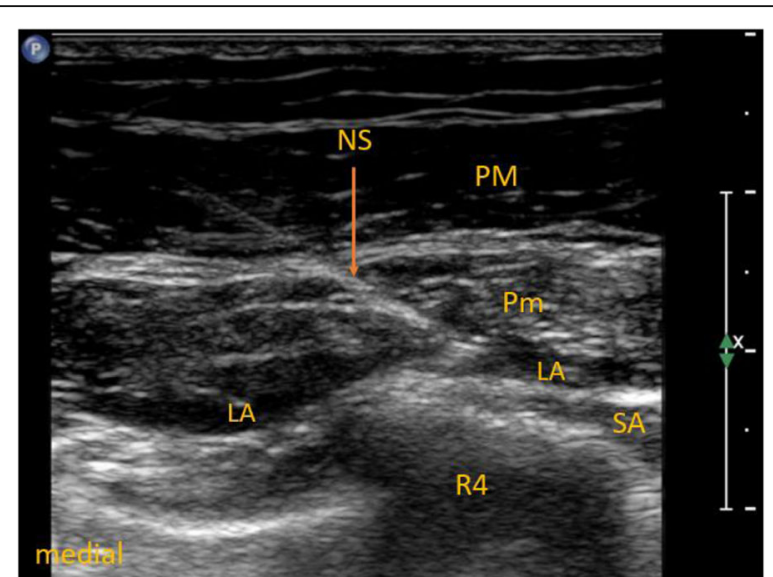

Fig. 3 Pectoralis nerves block type II. LA - local anesthetic, NS needle shaft, PM - pectoralis major muscle, Pm - pectoralis minor muscle, R4 - fourth rib, SA - serratus anterior muscle

bleeding and hemodynamic instability. If no problems were recognized, remifentanil infusion was discontinued, and the patient's trachea was extubated. Postoperative pain treatment was continued with a patient-controlled analgesia (PCA) pump which supplied oxycodone (1 $\mathrm{mg}$ per dose, at 7-min intervals, without basal infusion) during the first 24 postoperative hours.

Moreover, intravenous paracetamol, $1 \mathrm{~g}$ per $6 \mathrm{~h}$, was administered routinely. Postoperative pain was evaluated by nurses using the VAS at $2,4,6,8,12$, and $24 \mathrm{~h}$ postoperatively. Patients could evaluate their pain severity from 0 (no pain) to $100 \mathrm{~mm}$ (maximum pain) on the VAS. If pain intensity exceeding $40 \mathrm{~mm}$ on the VAS, up to two extra doses of oxycodone $(5 \mathrm{mg}$ each, rescue analgesia) could be administered intravenously by the nurse. Patients were transferred to the surgery ward by the end of the first postoperative day if no complications were present.

\section{Surgery}

For mini-invasive mitral and/or tricuspid valve surgery, the patient was placed in the supine position with elevated right hemithorax, and the right upper arm was flexed anteriorly with the forearm in front of the face. Transoesophageal echocardiographic (TEE) monitoring was performed for all patients to confirm the appropriate establishment of cardiopulmonary bypass (CPB), valvular repair, and heart de-airing. The chest was prepared and draped, and the right lung was deflated; a thoracotomy (5 to $7 \mathrm{~cm}$ in length) was then performed in the fourth intercostal space in the submammary fold, from the anterior to the medial axillary line. Small accessory incisions were made for the endoscope, aortic clamp, venting tube, $\mathrm{CO}_{2}$ line, and atrial retractor. 
$\mathrm{CPB}$ was established via femoral vessel cannulation; if tricuspid valve surgery was also planned, the right jugular vein was cannulated percutaneously. Patients were cooled to $34^{\circ} \mathrm{C}$, the pericardium was opened, and cardioplegia was administered to the aortic root after crossclamping of the aorta. The mitral and tricuspid valve (if required) was repaired using valvular rings and artificial Gore-Tex chordae, if required. After completion of the repair, patients were rewarmed and weaned from $\mathrm{CPB}$ and TEE examination was performed to assure the quality of the repair. The surgery site and the postoperative drain position are presented in Fig. 4.

\section{Outcomes}

\section{Primary outcome}

The total consumption of oxycodone during the first 24 postoperative hours. This outcome was presented also as morphine equivalence (ME, $1 \mathrm{mg}$ of oxycodone $=1.5 \mathrm{mg}$ of morphine [16]). Secondary outcome: Pain intensity assessed on the VAS at the $2,4,6,8,12$, and $24 \mathrm{~h}$ after surgery by nurses who were blinded to the type of treatment.

\section{Other outcomes}

The other measured variables were pain intensity (assessed by patients using the Prince Henry Hospital Pain Score (PHHPS)), patient satisfaction with pain management, and assessment of pulmonary function. PHHPS was used to assess the effect of analgesia provided by regional block and intravenously administered painkillers on deep breathing and coughing. Patients could describe their pain severity using a five-grade scoring system from 0 to 4 , in which 0 indicated 'no pain on coughing', 1 indicated 'pain

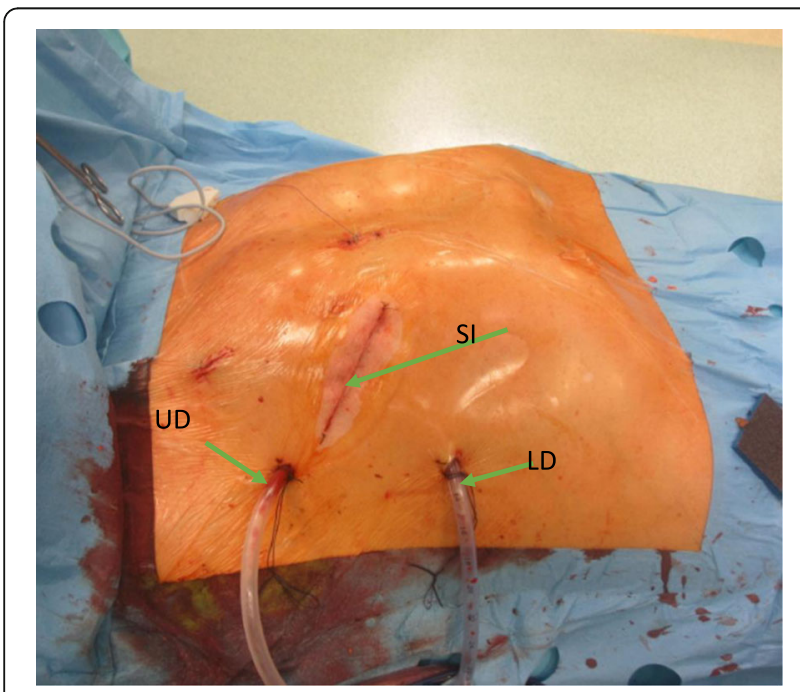

Fig. 4 Postoperative drain positions. The figure presents the positions of chest drains and the site of the incision. UD-upper drain, the proximal end in the apex of the lung, LD_lower drain, inserted horizontally ("lying on the diaphragm"), SI—surgical incision on coughing, but not on deep breathing', 2 indicated 'pain on deep breathing, but not at rest', 3 indicated 'slight pain at rest', and indicated 4 'severe pain at rest'. PHHPS was assessed at the time of admission, as well as at 1 day and 4 days after surgery. Patient satisfaction with pain management was assessed at the time of discharge from the hospital. Patients could describe their satisfaction with pain management as perfect (5), good (4), moderate (3), poor (2), or very poor (1).

Pulmonary function tests were performed by a physician who was not involved in anesthesia or surgery. The physician assessed each study participant by using the SP10W spirometer (Contec Medical Systems Co., Ltd., People's Republic of China) before surgery, as well as 1 day and 4 days after surgery.

\section{Statistical analysis}

Data are presented as medians [interquartile ranges (IQRs)]. The Mann-Whitney U test was used for nonparametric data. If normal distribution was confirmed, Student's t-test was used. Parametric data are presented as means with $95 \%$ confidence intervals (95\% CIs). All analyses were performed in Statistica 13.1 software (Stat Soft. Inc., Tulsa, OK, USA).

\section{Power analysis}

The sample size was calculated based on our preliminary results. The mean consumption of oxycodone was $22 \mathrm{mg}$ per day in patients who had the ESP block alone, and 10 mg in patients who had ESP, PECS I, and PECS II blocks. The calculated sample size was 12 individuals per group $(\alpha=0.05$; power $=0.8)$. Thus, we decided to recruit 15 patients in each group.

\section{Results}

This study was conducted from July 2018 to August 2018. Overall, 30 patients were analyzed, 15 per group (Fig. 5). Patient demographics and surgery times are presented in Table 1. No differences were found between the groups regarding patient demographics, surgery times, or American Society of Anesthesiologist Physical Status Classifications. We did not notice any relevant complications among the study participants.

\section{Oxycodone consumption}

The primary outcome of our study was the oxycodone consumption via PCA during the first 24 postoperative hours. Patients in the PECS + ESP group used significantly less oxycodone than individuals in the ESP group: 12 [IQR: 6-16] mg vs. 20 [IQR: $18-29$ ] mg or 18 [9-24] vs. 30 [27-43.5] ME ( $p=0.0004)$ (Fig. 6). Six patients required rescue dosages of oxycodone; all were in the ESP group. 


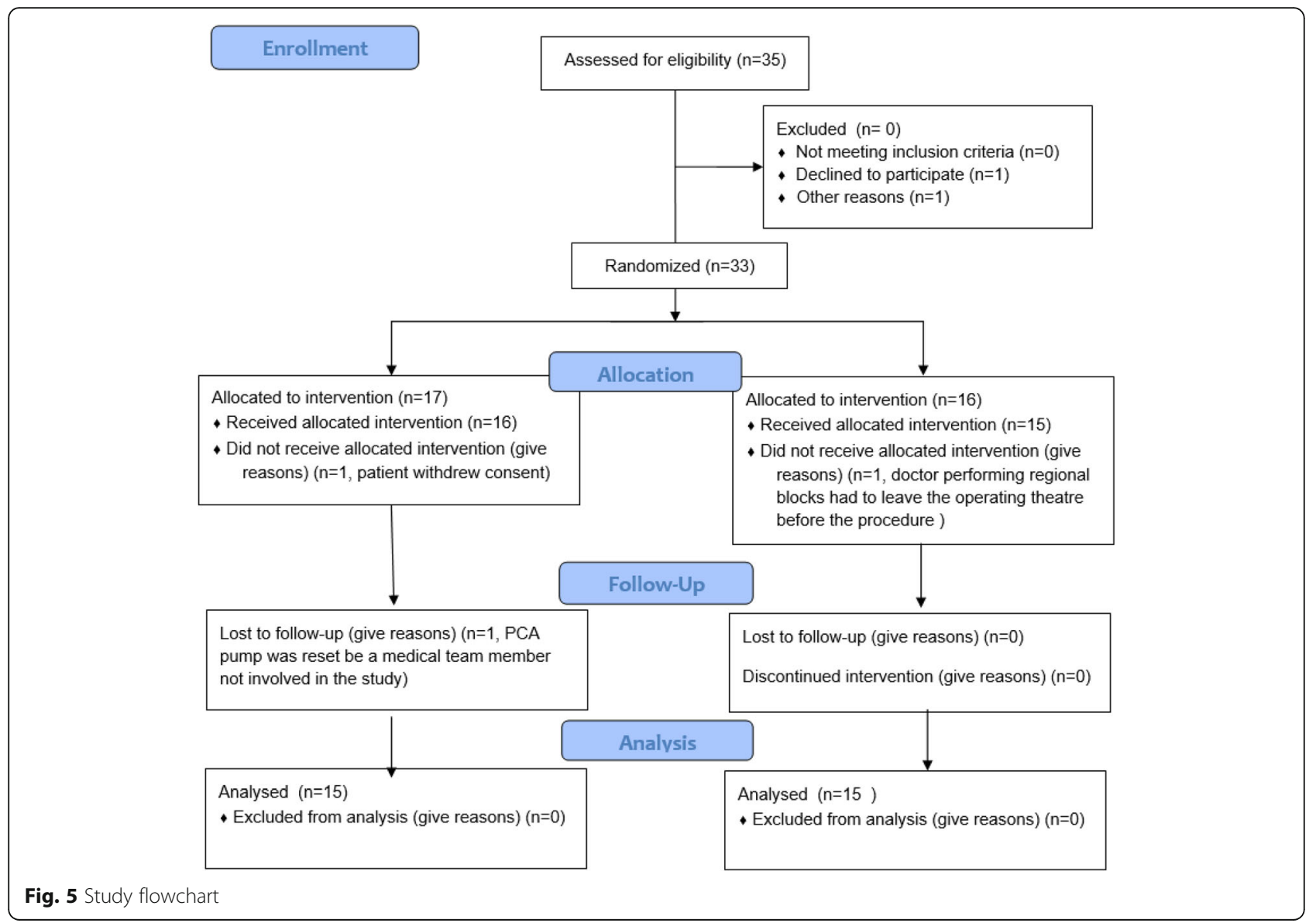

\section{Pain intensity}

Pain intensity was significantly lower in patients in the PECS group, compared with those in the ESP group, at the time of each clinical evaluation (Fig. 7, Table 2).

\section{Prince Henry hospital pain score}

No difference was found between the ESP and PECS + ESP groups regarding pain severity measured on PHHPS. None of the patients reported any pain at the time of admission. In both groups, pain severity was 1 [IQR: $1-1]$ on the first postoperative day and 1 [IQR: $0-$ $1]$ on the fourth postoperative day.

\section{Patient satisfaction with pain management}

Patients in the PECS + ESP group were more satisfied with pain management, compared with patients in the ESP group: 4 [IQR: 4-4] vs. 3 [IQR: $1-4]$ ] $(p=0.0007)$.

Table 1 Patient demographics

\begin{tabular}{llll}
\hline Group & ESP & PECS + ESP & $p$-value \\
\hline Age (years) & $60.7(53.9-67.6)$ & $53.9(45.7-62.0)$ & 0.18 \\
Weight $(\mathrm{kg})$ & $82.3(75.7-88.9)$ & $79.3(71.1-87.5)$ & 0.55 \\
Height $(\mathrm{m})$ & $175.5(170.5-180.5)$ & $172.6(167.1-178.1)$ & 0.41 \\
BMl $(\mathrm{kg} / \mathrm{m} 2)$ & $26.9(24.4-29.4)$ & $26.5(24.7-28.2)$ & 0.78 \\
Males N (\%) & $12(80)$ & $10(67)$ & 0.68 \\
Surgery time (minutes) & $226.7(207.3-246)$ & $213.7(189.5-237.8)$ & 0.38 \\
ASA & $2[2-2]$ & $2[2-2]$ & 0.63 \\
\hline
\end{tabular}

Age, weight, height, body mass index (BMI), and surgery time are shown as means and $95 \%$ confidence intervals. American Society of Anesthesiologists Physical Status Classification (ASA) is shown as median and interquartile range. Patient sex is shown as the number (percent) of males in each group. $P$-values were calculated with Student's t-test (normally distributed continuous data), the Mann-Whitney U test (non-normally distributed data), and the Fisher exact test (frequency data). ESP - erector spinae plane, PECS - pectoralis nerve 


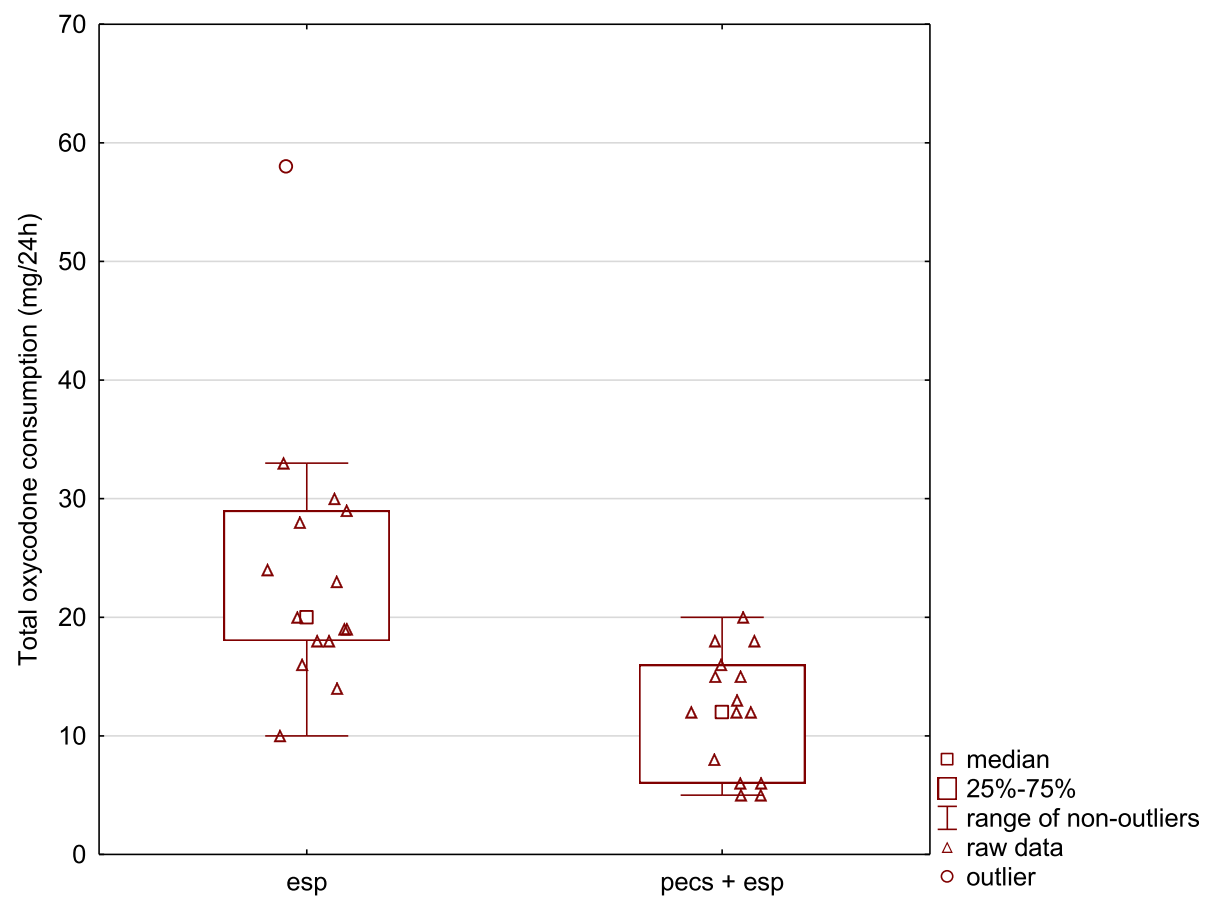

Fig. 6 Total oxycodone consumption during the first postoperative day was significantly lower in patients who had PECS I + PECS II + ESP block (PECS + ESP group) than in patients who had ESP block alone. Results are presented as medians and interquartile ranges. ESP - erector spinae plane, PECS - pectoralis nerve

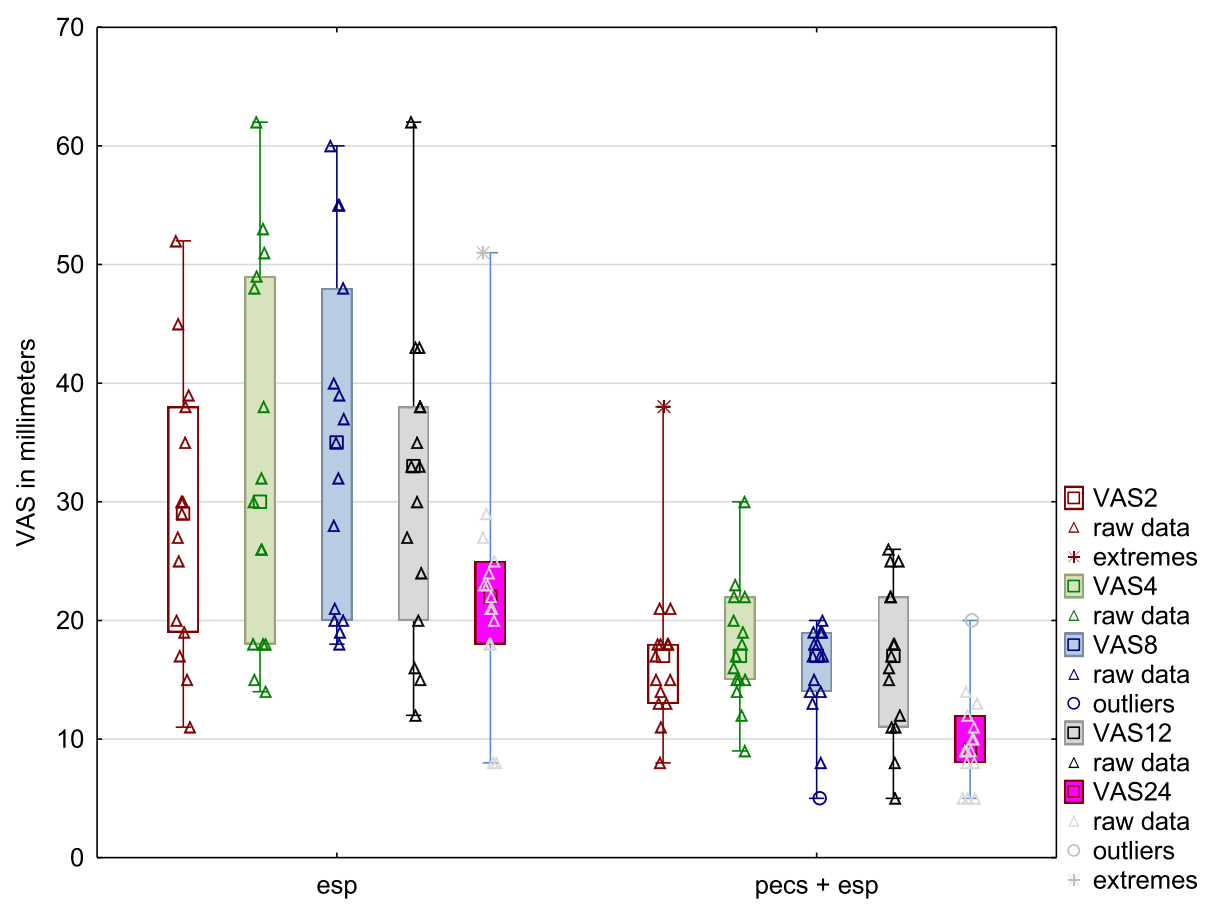

Fig. 7 Pain intensities reported by individual patients (triangles) and by groups of patients (boxes and whiskers) using the VAS. Results are presented as medians, 25th-75th percentile ranges (interquartile ranges - boxes), and 1st-99th percentile ranges (whiskers). VAS2, VAS4, VAS8, VAS12, and VAS24 denote pain intensity measurements at the second, fourth, eighth, 12th, and 24th hours postoperatively. ESP - erector spinae plane, PECS - pectoralis nerve, VAS - visual analog scale 
Table 2 Pain intensity

\begin{tabular}{llll}
\hline Pain evaluation & ESP & PECS + ESP & $p$ value \\
\hline $2 \mathrm{~h}$ & $29[19-38]$ & $17[13-18]$ & 0.004 \\
$4 \mathrm{~h}$ & $30[18-49]$ & $17[15-22]$ & 0.005 \\
$8 \mathrm{~h}$ & $35[18-49]$ & $17[14-19]$ & $<0.001$ \\
$12 \mathrm{~h}$ & $33[20-38]$ & $17[11-22]$ & 0.002 \\
$24 \mathrm{~h}$ & $22[18-25]$ & $9[8-12]$ & $<0.001$ \\
\hline
\end{tabular}

Pain intensity reported by patients and presented as medians and interquartile ranges. P-values were calculated with the Mann-Whitney U test. ESP - erector spinae plane, PECS - pectoralis nerve

\section{Pulmonary function tests}

Pulmonary function tests did not differ between the study groups for any of the evaluations. Selected parameters from pulmonary function tests are presented in Table 3. Pulmonary function decreased by approximately $30 \%$ from baseline but was similar in both groups.

\section{Discussion}

To our knowledge, this is the first randomized controlled trial (RCT) to compare ESP block with ESP plus PECS I and II blocks in patients undergoing cardiac surgery comprising valve surgery via right mini-thoracotomy. The results of the current study showed that the inclusion of an additional regional anesthesia technique (PECS I + PECS II blocks) with the ESP block significantly reduced oxycodone consumption and alleviated postoperative pain severity measured on the VAS (Figs. 6 and 7). Moreover, patients in the PECS + ESP group were more satisfied with pain management. However, pain management, as measured using the PHHPS, was good in both groups, and there was no difference in pulmonary function tests between the study groups. Of 30 patients, all could be weaned from mechanical ventilation in accordance with the study protocol (within $2 \mathrm{~h}$ from the end of the surgery).

ESP block provides satisfactory analgesia in patients after mini-thoracotomy procedures. In the current study, of 15 patients in the ESP group, 12 reported that their pain management was perfect or good; only a single participant reported pain management as poor. However, a continuing obstacle to the improvement of postoperative analgesia remains chest pain associated primarily with chest drains. We considered two regional techniques for additional analgesia: PECS and the serratus anterior block. Both methods have been described in patients who have undergone mini-thoracotomy procedures $[15,17]$. We chose to use PECS blocks due to our experience with this method. This modification significantly reduced postoperative pain and improved patient satisfaction in the PECS group.

Both ESP and PECS blocks are relatively new analgesic techniques. ESP is an interfascial plane block developed by Ferrero et al. in 2016 [18]. The deposition of local anesthetic in a location anterior to the erector spinae muscle causes multidermatomal sensory block on the ipsilateral side [19]. PECS blocks require an injection of local anesthetic into two planes: between the pectoralis major and pectoralis minor muscles; and between the pectoralis minor and serratus anterior muscles [15]. These techniques block branches of the brachial plexus (anterior thoracic nerves). Recently, new studies have shown further use of ESP and PECS block in cardiac surgery $[11,12]$.

Although PECS and ESP blocks appear to cover similar areas, their clinical efficacy is still under investigation. The results presented in cadaveric studies showed some unpredictably of ESP block $[19,20]$. In the study by Adhikary et al., the dye spread to the intercostal space was between 5 to 10 spaces, to the epidural space from 2 to 5 , and the intercostal foramina from 2 to 3 . Thus, the spread of dye in the ESP block was changeable and could differ significantly between only three cadavers. In a very recent study by Choi et al., 14 cadavers were evaluated (7 per group). Two volumes of dye were compared, 10 and $30 \mathrm{~mL}$. Similarly to the previous study, the dye was injected at the level of T5 [20].. Interestingly, the superior costotransverse ligament was stained in 3 of 7 cadavers at the level T3, and only in 1 of 7 cadavers at the T2 level after $30 \mathrm{~mL}$ of dye. In the current study, lower pain intensity and better patient satisfaction in the

Table 3 Pulmonary function tests

\begin{tabular}{|c|c|c|c|c|c|c|}
\hline \multirow[t]{2}{*}{ Time of assessment } & \multicolumn{3}{|l|}{ ESP } & \multicolumn{3}{|l|}{$\mathrm{PECS}+\mathrm{ESP}$} \\
\hline & $\overline{F V C ~(L) ~}$ & FEV1 (L/s) & $\operatorname{PEF}(\mathrm{L} / \mathrm{s})$ & $\overline{F V C ~(L) ~}$ & FEV1 (L/s) & $\operatorname{PEF}(\mathrm{L} / \mathrm{s})$ \\
\hline Admission & $3.3(2.5-4.0)$ & $2.7(2.1-3.3)$ & $6.4(4.9-7.9)$ & $3.4(3.0-3.8)$ & $2.7(2.5-3.0)$ & $5.6(4.8-6.4)$ \\
\hline$p$-value & 0.73 & 1.0 & 0.32 & & & \\
\hline POD1 & $2.3(1.8-2.9)$ & $1.8(1.4-2.2)$ & $4.7(3.7-5.6)$ & $2.6(2.2-3.0)$ & $2.0(1.7-2.3)$ & $4.8(3.9-5.7)$ \\
\hline$p$-value & 0.47 & 0.35 & 0.87 & & & \\
\hline POD4 & $2.6(2.1-3.1)$ & $2.1(1.7-2.5)$ & $6.2(5.1-7.3)$ & $2.8(2.4-3.2)$ & $2.2(1.8-2.6)$ & $5.4(4.4-6.3)$ \\
\hline$p$-value & 0.50 & 0.81 & 0.21 & & & \\
\hline
\end{tabular}

Selected results of pulmonary function tests in both groups of patients. Spirometry was performed 1 day before surgery (admission), 1 day after surgery (POD1), and 4 days after surgery (POD4). Data are presented as means and 95\% confidence intervals. P-values were calculated with Student's t-test was. ESP - erector spinae plane, PECS - pectoralis nerve, FVC - forced vital capacity, FEV1 - forced expiratory volume in $1 \mathrm{~s}$, PEF - peak expiratory flow 
PECS + ESP group could be caused by the covering area not fully supplied by ESP block in some patients. It appears that pain intensity alleviation and improved patient satisfaction could be caused by only PECS II block. PECS I block which covers a small area of the anterior chest wall could be an unnecessary procedure in our trial. However, we cannot fully exclude its usefulness in this case. More evidence is necessary.

Other potential techniques that could be used in patients after mitral and/or tricuspid valve repair via minithoracotomy include PVB and TEA. PVB seems superior to TEA for this type of surgery because its analgesic area is limited to the operated side $[1,21]$. Data to compare pain relief between ESP and PVB are lacking, but we suspect that their efficacy is similar. However, we hypothesized that PVB could be associated with an increased risk of pleural puncture, relative to that of ESP block [22]. Further RCTs are needed to investigate whether ESP and PVB are equivalent with respect to pain management, complication rate, and patient satisfaction.

The other regional anesthesia method which could be effective after mini-thoracotomy procedures are the intercostal blockade. This procedure could be performed at the end of surgery by the surgeon under direct vision. However, the intercostal blockade provides the highest plasma ropivacaine concentration of all anesthetic techniques, with the peak plasma concentration at $21 \pm 9$ min from injection and sensory blockade (measured by pinprick) lasting of $6.0 \pm 2.5 \mathrm{~h}$ only [23].

Our study had some limitations. Although statistical significance was demonstrated for primary and secondary outcomes, the sample size was relatively small. Thus, the lack of complications could be the result of a low number of participants. The current study showed that the addition of PECS block to ESP block improved postoperative pain control and increased patient satisfaction. However, PECS blocks may be sufficient as a single regional analgesia technique for pain management in patients undergoing valve repair via right mini-thoracotomy. Moreover, PECS blocks could be superior to ESP block for this type of surgery. The current study did not exclude this alternative. Neither ESP nor PECS blocks effectiveness was confirmed in the operating theatre with the loss of sensation technique before the surgery.

\section{Conclusion}

In conclusion, the current study demonstrated that the addition of PECS blocks to ESP block led to reduced consumption of oxycodone via PCA, reduced pain intensity on VAS, and increased patient satisfaction with pain management in patients undergoing mitral/tricuspid valve repair via mini-thoracotomy. However, there were no differences between the study groups regarding pulmonary function tests.

\section{Abbreviations}

CBP: Cardiopulmonary bypass; Cl: Confidence interval; ERAS: Enhanced Recovery After Surgery; ESP: Erector spinae plane; IQR: Interquartile range; ME: Morphine equivalence; PCA: Patient-controlled analgesia; PECS: Pectoralis nerve; PHHPS: Prince Henry Hospital Pain Score; PVB: Paravertebral block; TEA: Thoracic epidural analgesia; TEE: Transoesophageal echocardiography; VAS: Visual analog scale

\section{Acknowledgments}

None.

\section{Authors' contributions}

$B G, M B, B B, J B, M C, B W G, M K, K W$ : conceived and designed the study. $B G, B B$, $M K, K W$ : conducted the study. MB, BG, BWG: analyzed the data. MB, BWG, and BG: prepared the first draft of the manuscript. BB, JB, MC, MK, KW: contributed to the major revision of the manuscript. All authors contributed to the final manuscript revisions and approved the final version.

\section{Funding}

None.

\section{Availability of data and materials}

The datasets used and/or analyzed during the current study are available from the corresponding author on reasonable request.

\section{Ethics approval and consent to participate}

Approved by the Bioethics Committee of the Medical University of Lublin, Lublin, Poland (permit number KE-0254/127/2018), and registered at ClinicalTrials.gov (NCT03592485). Written informed consent was obtained from each patient.

\section{Consent for publication}

Not applicable.

\section{Competing interests}

The authors declare that they have no competing interests.

\section{Author details}

${ }^{1}$ Division of Cardiovascular Surgery, St. Jadwiga Provincial Clinical Hospital, ul. Lwowska 60, 35-301 Rzeszów, Poland. ²Second Department of Anesthesia and Intensive Care, Medical University of Lublin, ul. Staszica 16, 20-081 Lublin, Poland. ${ }^{3}$ Anesthesiology and Intensive Care Department with the Center for Acute Poisoning, St. Jadwiga Provincial Clinical Hospital, ul. Lwowska 60, 35-301 Rzeszów, Poland.

Received: 4 October 2019 Accepted: 17 February 2020

Published online: 27 February 2020

\section{References}

1. Mesbah A, Yeung J, Gao F. Pain after thoracotomy. BJA Education. 2016; 16(1):1-7. https://doi.org/10.1093/bjaceaccp/mkv005.

2. Agostini $P$, Cieslik H, Rathinam $S$, et al. Postoperative pulmonary complications following thoracic surgery: are there any modifiable risk factors? Thorax. 2010;65:815-8.

3. Szelkowski LA, Puri NK, Singh R, Massimiano PS. Current trends in preoperative, intraoperative, and postoperative care of the adult cardiac surgery patient. Curr Probl Surg. 2015;52:531-69. https://doi.org/10.1067/j. cpsurg.2014.10.001 Epub 2014 Oct 28.

4. Noss C, Prusinkiewicz C, Nelson G, Patel PA, Augoustides JG, Gregory AJ. Enhanced Recovery for Cardiac Surgery. J Cardiothorac Vasc Anesth. 2018; S1053-0770(18):30049-1. https://doi.org/10.1053/j.jvca.2018.01.045.

5. Landoni G, Isella F, Greco M, Zangrillo A, Royse CF. Benefits and risks of epidural analgesia in cardiac surgery. Br J Anaesth. 2015;115:25-32. https:// doi.org/10.1093/bja/aev201.

6. Scott NB, Turfrey DJ, Ray DA, Nzewi O, Sutcliffe NP, Lal AB, Norrie J, Nagels WJ, Ramayya GP. A prospective randomized study of the potential benefits of thoracic epidural anesthesia and analgesia in patients undergoing coronary artery bypass grafting. Anesth Analg. 2001;93(3):528-35.

7. Bignami E, Landoni G, Biondi-Zoccai GG, et al. Epidural analgesia improves outcome in cardiac surgery: a meta-analysis of randomized controlled trials. J Cardiothorac Vasc Anesth. 2010;24:586-97. 
8. Scarfe AJ, Schuhmann-Hingel S, Duncan JK, Ma N, Atukorale YN, Alun L. Cameron; Continuous paravertebral block for post-cardiothoracic surgery analgesia: a systematic review and meta-analysis. Eur J Cardio-Thoracic Surg. 2016;50(6):1010-8. https://doi.org/10.1093/ejcts/ezw168.

9. Tahara $\mathrm{S}$, Inoue A, Sakamoto $\mathrm{H}$, et al. A case series of continuous paravertebral block in minimally invasive cardiac surgery. JA Clin Rep. 2017; 3:45.

10. Chakravarthy M. Regional analgesia in cardiothoracic surgery: a changing paradigm toward opioid-free anesthesia? Ann Card Anaesth. 2018;21:225-7.

11. Kumar KN, Kalyane RN, Singh NG, Nagaraja PS, Krishna M, Babu B, Varadaraju R, Sathish N, Manjunatha N. Efficacy of bilateral pectoralis nerve block for ultrafast tracking and postoperative pain management in cardiac surgery. Ann Card Anaesth. 2018;21:333-8.

12. Nagaraja PS, Ragavendran S, Singh NG, Asai O, Bhavya G, Manjunath N. Rajesh $\mathrm{K}$ comparison of continuous thoracic epidural analgesia with bilateral erector spinae plane block for perioperative pain management in cardiac surgery. Ann Card Anaesth. 2018;21:323-7.

13. Borys M, Gawęda B, Horeczy B, et al. Erector spinae-plane block as an analgesic alternative in patients undergoing mitral and/or tricuspid valve repair through a right mini-thoracotomy - an observational cohort study. Videosurgery and Other Miniinvasive Techniques/Wideochirurgia i inne techniki małoinwazyjne. 2019. https://doi.org/10.5114/wiitm.2019.85396.

14. Blanco R. The 'pecs block': a novel technique for providing analgaesia after breast surgery. Anesthesia. 2011;66:847-8.

15. Yalamuri S, Klinger RY, Bullock WM, Glower DD, Bottiger BA, Gadsden JC. Pectoral Fascial (PECS) I and II blocks as rescue analgesia in a patient undergoing minimally invasive cardiac surgery. Reg Anesth Pain Med. 2017; 42:764-6. https://doi.org/10.1097/AAP.0000000000000661.

16. Kalso E, Pöyhiä R, Onnela P, Linko K, Tigerstedt I, Tammisto T. Intravenous morphine and oxycodone for pain after abdominal surgery. Acta Anaesthesiol Scand. 1991;35:642-6. https://doi.org/10.1111/j.1399-6576.1991. tb03364.

17. Costa F, Nenna A, Barbato R, Benedetto M, Del Buono R, Agrò FE. Serratus anterior plane block for right minithoracotomy revision after mitral valve repair. Minerva Anestesiol. 2017;83:1333-4. https://doi.org/10.23736/S03759393.17.12186-3.

18. Forero M, Adhikary SD, Lopez H, et al. The erector Spinae plane block: a novel analgesic technique in thoracic neuropathic pain. Reg Anesth Pain Med. 2016;41:621-7.

19. Adhikary S, Bernard S, Lopez H, et al. Erector Spinae Plane Block Versus RetrolaminarBlock: A Magnetic Resonance Imaging and Anatomical Study. Reg Anesth Pain Med. 2018;23:756-62. https://doi.org/10.1097/AAP. 0000000000000798.

20. Choi YJ, Kwon HJ, O J, et al. Influence of injectate volume on the paravertebral spread in erector spinae plane block: An endoscopic and anatomical evaluation. PLoS One. 2019;14:e0224487. https://doi.org/10.1371/ journal.pone.022448.

21. Carmona P, Llaqunes J, Casanova I, Mateo E, Cánovas S, Martín E, Marqués $\mathrm{Jl}$, Peña JJ, de Andrés J. Continuous paravertebral analgesia versus intravenous analgesia in minimally invasive cardiac surgery by minithoracotomy. Rev Esp Anestesiol Reanim. 2012;59:476-82. https://doi.org/10. 1016/j.redar.2012.04.014.

22. Kus A, Gurkan Y, Gul Akgul A, Solak M, Toker K. Pleural puncture and intrathoracic catheter placement during ultrasound guided paravertebral block. J Cardiothorac Vasc Anesth. 2013;27:e11-2. https://doi.org/10.1053/j. jvca.2012.10.018.

23. Kopacz DJ, Emmanuelsson BM, Thompson GE, et al. Pharmacokinetics of ropivacaine and bupivacaine for bilateral intercostal blockade in healthy male volunteers. Anesthesiology. 1994:81:1139-48. https://doi.org/10.1097/ 00000542 .

\section{Publisher's Note}

Springer Nature remains neutral with regard to jurisdictional claims in published maps and institutional affiliations.

Ready to submit your research? Choose BMC and benefit from:

- fast, convenient online submission

- thorough peer review by experienced researchers in your field

- rapid publication on acceptance

- support for research data, including large and complex data types

- gold Open Access which fosters wider collaboration and increased citations

- maximum visibility for your research: over $100 \mathrm{M}$ website views per year

At BMC, research is always in progress.

Learn more biomedcentral.com/submissions 